\title{
An analysis of intraoperative blood transfusion of cardiac surgery in one hospital from 2014 to 2016
}

\author{
Sihua Yi ${ }^{*}$, Cuicui Chai, Jing Lin, Hongliang Zhang, Qiangjun Luo,Han Wang, Jiangbo Chen, Dan Li \\ Department of Blood Transfusion, the First Hospital of Lanzhou University, Lanzhou 730000, China.
}

\begin{abstract}
To investigate the usage of blood componentsfor cardiac surgery inthe First Hospital of Lanzhou University, data from January 2014 to December 2016 were collected for analysis, including the number of cardiac surgeries and blood transfusions. There were 1589 males and 1076 females, aged from 1 to 73 years, with an average age of $(53.97 \pm 11.33)$ years, in this study. The results showed that the rate of blood-free surgery in the hospital increased year by year,while the proportion of blood-used operation to total operations decreased every year. The ratio of plasma and platelets used in cardiac surgery decreased, while the cryoprecipitate ratio used in cardiac surgery increased. This study indicates that the transfusion medical level in cardiac surgery is improved continuously, and that the infusion of blood components has become more secure, efficient and rational.
\end{abstract}

Keywords: intraoperative blood transfusion; cardiac surgery; blood component transfusion

\section{INTRODUCTION}

As an important means of clinical treatment, blood transfusions are widely carried out inclinics throughout the world. For patients undergoing cardiac surgery, the adequate and timely supply of blood components is one of the main factors ensuring an operation success. However, blood transfusion is also a risky treatment, which can infect the patient with serious diseases such as AIDS, syphilis or hepatitis if not handled appropriately. For this reason, the World Health Organization (WHO) emphasized:"The rational clinical use of blood, and use of blood substitutes as much as possible, in order to minimize unnecessary blood transfusions." Compared with other blood components, plasma infusion is more dangerous, in some cases causing complications such as transfusionrelated acute lung injury (TRALI), transfusion-related circu- latory overload, allergic reactions and other adverse transfusion reactions ${ }^{[1]}$. Unfortunately, plasma usage is higher when compared with cryoprecipitate, especially during cardiac surgery in China.

In recent years, with the development of new blood transfusion techniques, such as thromb elastography (TEG) and the improved management of patient blood usage ${ }^{[2]}$, blood component transfusion has become more and more widespread compared to whole blood transfusion. However, there are still some misunderstandings in the application of plasma, such as using plasma to supplement blood volume and albumin, and enhance immunity. As target has been set for plasma use ratio, it has become an important marker for the rational use of blood in a hospital and even a region. As a consequence, a widespread decrease in clinical plasma used in blood transfusion, and an increase of platelet and cryoprecipitation transfusion indicate that

*Correspondence to: Sihua Yi, Department of Blood Transfusion, the First Hospital of Lanzhou University, No.11 Donggang West Road, Lanzhou, Gansu730000, China. E-mail: ldyysxk@163.com.

Conflict of interests: The authors declared no conflict of interests. 
the levels of rational blood use in clinics are improving $^{[3]}$. This study was conducted in the First Hospital of Lanzhou University to investigate the rational use of blood for cardiac surgery by analyzing the ratio of blood-free surgery, the change of average blood volume per operation and the proportion of each blood component transfusion in cardiac surgery over recent three years.

\section{MATERIALS AND METHODS}

\section{Clinical data collection}

A total of 2665 patients who underwent cardiac surgery at the First Hospital of Lanzhou University from January 2014 to December 2016 were selected, including 1589 males and 1076 females, aged from 1 to 73 years, with an average age of $(53.97 \pm 11.33)$ years. The surgery included ductus arteriosus, mitral and aortic valve related surgeries, tetralogy of Fallot, aortic dissection and other cardiac surgeries. The data, including the number of cardiac surgeries and blood transfusions were obtained from records at the First Hospital of Lanzhou University from 2014 to 2016. This study was performed with the approval of Ethics Committee of the hospital.

\section{Intraoperative blood transfusion}

All cardiac surgeries including blood-free operations, extracorporeal anesthesia blood transfusions and the usage of red blood cells, plasma, platelets and cryoprecipitate in extracorporeal anesthesia blood transfusion from 2014 to 2016 were tabulated. The red blood cells included red cells suspension, suspended leukocyte-reduced red blood cells and washed red blood cells, while the plasma included frozen plasma (FP), fresh frozen plasma (FFP) and virus inactivated plasma.

The blood volume was calculated in unit (U). The red blood cells were directly calculated according to the unit labeled in the blood bag (the quantity obtained from $200 \mathrm{~mL}$ whole blood separation was set as one unit), $200 \mathrm{~mL}$ of plasma was calculated as one unit, one therapeutic dose of apheresis platelets was calculated as one unit, and the cryoprecipitate prepared from $200 \mathrm{~mL}$ whole blood was also calculated as one unit. The percentage of blood components = usage of each component $(\mathrm{mL})$ / sum of blood components $(\mathrm{mL})$.

\section{Statistical analysis}

The ratio of blood-free operations and the ratio of blood components used in cardiac surgery were statistically analyzed by theMann-Whitney U test, and the mean blood volume per operation was statistically analyzed by the t test. $P<0.05$ was considered statistically significant.

\section{RESULTS}

\section{The rate of blood-free surgery increased in cardiac surgery from 2014 to 2016}

The proportion of blood-free surgery to total operations increased each year from 2014 to 2016, while the proportion of blood-used operation to total operations decreased each year. In 2014, from a total operation volume of 796, there were 255 cases(32.04\%) of blood-free surgery and 541 cases $(67.96 \%)$ requiring blood transfusion. In 2015, from a total operation volume of 890 , there were 327 cases $(36.74 \%)$ of blood-free surgery and 563 cases $(63.26 \%)$ requiring blood transfusion. In 2016, from a total of 979 operations, there were 408 cases $(41.68 \%)$ of blood-free surgery and 571 operations $(58.32 \%)$ requiring blood transfusion(Table 1).

Table 1 Analysis of the ratio of blood-free surgery to total operation volume in cardiac surgery from 2014 to 2016

\begin{tabular}{|c|c|c|c|}
\hline Sugery methods & 2014 & 2015 & 2016 \\
\hline Blood transfusion operation & $541(67.96)$ & $563(63.26)^{*}$ & $571(58.32)^{* \Delta}$ \\
\hline Blood-free operation & $255(32.04)$ & $327(36.74)^{*}$ & $408(41.68) * \Delta$ \\
\hline Total operation volume & 796 & 890 & 979 \\
\hline
\end{tabular}

The ratio of plasma and apheresis platelets decreased during cardiac surgery from 2014 to 2016

From 2014 to 2016, the ratio of intraoperative plasma and apheresis platelet transfusions to total blood volume decreased every year with significance, while the ratio of intraoperative cryoprecipitate transfusions increased year by year,which was also significant. The percentages of plasma to total blood volume during cardiac surgery from 2014 to 2016 were $44.02 \%$, $33.40 \%$ and $24.69 \%$, respectively. The percentages of apheresis platelet to total blood volume during cardiac surgery from 2014 to 2016 were $3.86 \%, 3.60 \%$ and $3.36 \%$, respectively. The percentage of cryoprecipitate to total blood volume during cardiac surgery from 2014 to 2016 was $34.32 \%, 48.46 \%$ and $57.89 \%$, respectively(Table 2$)$.

The average blood transfusion volume per operation decreased during cardiacsurgery in 2015

A total of 819 cardiac surgeries were performed in 2014, and the average blood transfusion volume used in each operation was $(7.16 \pm 1.56) \mathrm{U}$. A total of 857 
Table 2 Analysis of intraoperative blood component transfusion in cardiac surgery from 2014 to 2016

\begin{tabular}{|c|c|c|c|c|c|c|c|c|c|}
\hline \multirow{2}{*}{ Year } & \multicolumn{2}{|c|}{ Red blood cells } & \multicolumn{2}{|c|}{ Plasma } & \multicolumn{2}{|c|}{ Apheresis platelets } & \multicolumn{2}{|c|}{ Cryoprecipitation } & \multirow{2}{*}{$\begin{array}{c}\text { Total } \\
\text { volume(U) }\end{array}$} \\
\hline & Usage(U) & Percentage $(\%)$ & Usage(U) & Percentage (\%) & Usage(U) & Percentage (\%) & Usage(U) & Percentage (\%) & \\
\hline 2014 & 1044 & 17.80 & 2582.5 & 44.02 & 226.5 & 3.86 & 2013.5 & 34.32 & 5866.5 \\
\hline 2015 & 782 & $14.54^{*}$ & 1796.5 & $33.40^{*}$ & 193.5 & $3.60^{*}$ & 2606.5 & $48.46^{*}$ & 5378.5 \\
\hline 2016 & 905.5 & $14.07^{*}, \Delta$ & 1588.5 & $24.69^{*, \Delta}$ & 216.0 & $3.36^{*}, \Delta$ & 3725 & $57.89^{*, 4}$ & 6435 \\
\hline
\end{tabular}

Note: compared with the datain $2014,{ }^{*} P<0.05$; compared with the data in $2015,{ }^{\triangle} P<0.05$

cardiac surgeries were performed in 2015, and the average blood transfusion volume used in each operation was $(6.28 \pm 1.23) \mathrm{U}$, which was lower than that in 2014 with significant difference $(P<0.05)$. A total of 916 cardiac operations were performed in 2016, with anaverage blood transfusion volume of $(7.03 \pm 0.98)$ U per operation, which was lower than that in 2014 with no significant difference $(P>0.05)$, and which although higher than that in 2015 ,the difference was not significant $(P>0.05$, Table 3).

Table 3 The average blood transfusion volume per operation in cardiac surgery from 2014 to 2016

\begin{tabular}{lccc}
\hline \multicolumn{1}{c}{ Index } & 2014 & 2015 & 2016 \\
\hline Total blood volume in cardiac & 5866.5 & 5378.5 & 6435 \\
surgery(U) & & & \\
Total number of cardiac surgery & 819 & 857 & 916 \\
Average blood transfusion & $7.16 \pm 1.56$ & $6.28 \pm 1.23^{*}$ & $7.03 \pm 0.98$ \\
volume per operation(U) & & & \\
\hline
\end{tabular}

Note: compared with the average blood transfusion volume per operation in $2014,{ }^{*} P<0.05$. There was no significant difference in the average blood volume per operation between 2016 and $2015(P=0.5730)$.

\section{DISCUSSION}

The purpose of modern clinical blood transfusion service is to increase the security, efficiency, rationality and economy of every clinical blood transfusion ${ }^{[4]}$. The WHO's Blood Safety and Clinical Technology Srategy states that promoting appropriate clinical usage of blood and preventing unnecessary transfusions are important measures to ensure blood transfusion safety ${ }^{[5-6]}$. Therefore, in the process of blood transfusion treatment, the correct selection and effective provision of blood components are extremely important for the efficient and safe treatment for patients.

From 2014 to 2016 , the proportion of blood-free operation to the total operation volume of cardiac surgery in the First Hospital of Lanzhou University was $32.04 \%, 36.74 \%$, and $41.68 \%$,respectively. The increase in the rate of blood-free surgery each year reflected the continuous improvements of the surgical technical level and precision of cardiac surgeons in our hospital, through continuous learning and summing up experience, which also reflected the cardiac surgeons'increased risk awareness, aiming for"Less blood transfusion, no blood transfusion."Cardiac surgeons actively promoted the recycled autologous blood transfusion technology to patients and their families, which reduced the rate of allogeneic blood transfusions. In order to do this, clinicians should follow strictly blood transfusion indication in blood transfusion assessments, reduce and even eliminate preventive blood transfusions, avoid matching blood transfusion, comforting blood transfusion and nutritional blood transfusion. A more comprehensive evaluation of clinical blood transfusions should be carried out by combining the disease conditions with laboratory examinations, which should eventually reduce the total amount of intraoperative allogeneic blood transfusions.

The average blood transfusion volume used for each operation in 2015 was $(6.28 \pm 1.23) \mathrm{U}$, which was significantly lower than that of $(7.16 \pm 1.56) \mathrm{U}$ in 2014 . However, the average blood transfusion volume per operation $(7.03 \pm 0.98) \mathrm{U}$ in 2016 was not decreased compared with that in 2015 , but was increased, mainly due to the increase of intraoperative cryoprecipitation in 2016. The utilization rate of cryoprecipitate was increased from $48.46 \%$ in 2015 to $57.89 \%$ in 2016 , which indicated that cardiac surgeons in our hospital gradually recognized the efficacy of cryoprecipitation. From 2014 to 2016, the ratio of intraoperative plasma transfusion to the total blood volume decreased year by year,which was $44.02 \%, 33.40 \%, 24.69 \%$, respectively. The decrease in utilization rate of plasma and the increase in utilization rate of cryoprecipitate reflected improvements in blood transfusion expertise, the use of blood components, the consciousness of reasonable and effective use of blood. The medical level of clinical blood transfusion has been continuously improved among cardiac surgeons in our hospital by continuous safety training for clinical blood use, popularizing new concepts and techniques of blood transfusion. The understanding and execution of rational blood use by cardiac surgeons in our hospital have been strengthened. The pre-infusion assessment of plasma has changed from focusing solelyon coagulation results to combining clinical bleeding and other coagulation indexes such as platelet count. For example, TEG was used to predict clinical blood use or correct blood use deviation, and then a more appropriate blood transfusion strategy was chosen ${ }^{[7]}$. In 
summary, clinicians involved in implementing blood transfusion strategies at the First Hospital of Lanzhou University have improved their understanding of the curative effects of platelet and cryoprecipitation based on related indications ${ }^{[8]}$, while their patients have gained access to more reasonable infusions of red blood cells, plasma, platelets, and cryoprecipitation.

To improve the level of clinical rational use of blood is a systematic project. Relevant measures should be taken in the practice of blood transfusion to improve the level of rational and scientific blood use ${ }^{[9-10]}$. The rational use of blood in clinic is ultimately reflected in the actual diagnosis and treatment process of clinicians. Only when clinicians make correct decisions on blood transfusion and choose the variety and dosage of blood products properly, can the curative effects of blood transfusion meet expectations ${ }^{[7,11]}$. Therefore, in order to fundamentally improve the medical level of blood transfusion, it is necessary to strengthen all training regarding the rational use of blood for clinical medical staff ${ }^{[9]}$. The blood transfusion department should actively propagandize blood transfusion knowledge, in order to inform clinicians on how to choose blood products correctly, such as by training clinicians on the "Scientific and rational use of blood" every year ${ }^{[12]}$, and providing in-depth introduction on the function of various blood products ${ }^{[13]}$. In addition, transfusion technicians should actively communicate with clinicians and flexibly control suspected unreasonable blood transfusions. At the same time, a rational blood use evaluation system should be established and unreasonable blood transfusions should be standardized $^{[14]}$. The hospitals should establish routine post-transfusion evaluation strategies to promote continuous increases in the proportion of rational blood use $\mathrm{e}^{[13]}$.

\section{References}

[1] Guo X, Kuai DW. Review on the research progress of clinical blood transfusion[J]. International Journal of Laboratory Medicine(In Chinese), 2014, 35(18) :24992502

[2] Yue L, Zhao J. Progress in the application of throm- boelastography in malignant tumor-associated venous thromboembolism[J]. Journal of Modern Clinical Medicine(In Chinese), 2019, 45(4): 317-320

[3] Tao CH, Turu. Statistical analysis of clinical blood rransfusion in our hospital from 2000 to 2007[J]. Journal of Clinical Hematology(In Chinese), 2009, 22(4): 208-210

[4] Zhong Chunping, ShenWeidong, Li B, et al. Application of clinical blood transfusion services in transfusion therapy[J]. Modern Medical Journal(In Chinese), 2011, 11(1): 122-124

[5] Wu WJ, Tian ZS. Basic procedures to be followed in clinical blood transfusion[J]. Chinese Journal of Blood Transfusion(In Chinese), 2008, 21(3): 226-231

[6] WHO, Blood safety and clinical technology: Strategy 2000-2003[M]. Geneva:WHO, 2001: 2-3

[7] Desborough M, Stanworth S. Plasma transfusion for bedside, radiologically guided, and operating room invasive procedures[J]. Transfusion, 2012, 52(1, SI): 20S-29S

[8] Huang JY, Wei YM, Hu YH, et al. Establishment of assessment system of blood transfusion application and case medical record for clinic transfusion monitor[J]. Chinese Journal of Blood Transfusion(In Chinese), 2012, 25(10): 1059-1062

[9] Yang BC, Shao CP. Thoughts on promoting the rational use of blood in clinical practice[J]. Chinese Journal of Blood Transfusion(In Chinese), 2009, 22(5): 411-413

[10] Zhang YZ, Zhang H, Meng QB, et al. The necessity and solution of standardized management of clinical blood transfusion[J]. Chinese Journal of Blood Transfusion(In Chinese), 2008, 21(3): 214-215

[11] Yang BC, Zhang YZ. Management of blood collection, supply and clinical blood transfusion[M].Beijing:people's medical publishing house, 2011: 165-176

[12] Deng MY, Chen Y, Su ML, et al. The role of blood transfusion branch in hospital's clinical transfusion management[J]. China Medical Herald(In Chinese), 2009, 6(31): 159-160

[13] Meng QB. Some problems and solving strategies in clinical blood transfusion management $[\mathrm{J}]$. Chinese Journal of Blood Transfusion(In Chinese), 2010, 23(9): 734-736

[14] Tu XH, ZangY, Gu HH, et al. Perioperative blood transfusion is associated with poorer survival in patients with gastric cancer[J]. Asia-Pacific Journal of Blood Types and Genes, 2017, 1(2): 7-13

(Received 26 August 2019, Revised 7 December 2019, Accepted 15 December 2019) 